\title{
Regional differences in prognostic value of cardiac valve plane displacement in systemic light-chain amyloidosis
}

Marco M. Ochs ${ }^{1 *}$, Thomas Fritz ${ }^{1}$, Nisha Arenja ${ }^{1}$, Johannes Riffel ${ }^{1}$, Florian Andre', Derliz Mereles', Fabian aus dem Siepen ${ }^{1}$, Ute Hegenbart ${ }^{2}$, Stefan Schönland ${ }^{2}$, Hugo A. Katus ${ }^{1}$, Matthias G. W. Friedrich ${ }^{1}$ and Sebastian J. Buss ${ }^{1}$

\begin{abstract}
Background: To compare the prognostic value of cardiac valve plane displacement (CVPD) on various locations in cardiac light chain (AL) amyloidosis.

Methods: Consecutive patients with biopsy-proven cardiac involvement in AL amyloidosis who had undergone cardiovascular magnetic resonance (CMR) between 2005 and 2014 in our institution, were retrospectively identified and data analyzed. The primary combined endpoint was all-cause mortality or heart transplantation. Systolic CVPD were obtained from standard cine bSSFP in 2-, 3- and 4-chamber views at anterior aortic plane systolic excursion (AAPSE); anterior, anterolateral, inferolateral, inferior, inferoseptal mitral (MAPSE); and lateral tricuspid (TAPSE) annular segments.

Results: We identified 68 patients (58 \pm 10 years; 59\% male). Median follow-up period was 1.2 years (IQR, 0.3-4.1). Significant differences in CVPD between patients who reached a primary endpoint $(n=44)$ and transplant-free survivors were found only for AAPSE (6.1 mm (IQR, 4.6-9.4) vs. $8.8 \mathrm{~mm}(\mathrm{IQR}, 6.9-10.4) ; p=0.02)$ and MAPSE $\mathrm{anterolateral}_{\text {. }}$ (7.3 mm (IQR, 5.4-11.7) vs. $10.5 \mathrm{~mm}(\mathrm{IQR}, 8.1-13.4) ; p=0.03)$. AAPSE $\left(X^{2}=15.6 ; p=0.0002\right)$ provided the best predictive value for transplant-free survival compared to all other valvular plane locations. A high-risk cutoff (AAPSE $\leq 7.6 \mathrm{~mm}$ ) was calculated by ROC analysis to predict all-cause death or heart transplantation within 6 months from index examination (AUC $=0.80 ; \mathrm{Cl}$ : 0.68 to 0.89; $p<0.0001$ ). AAPSE added incremental prognostic power to an imaging prediction model of late gadolinium enhancement and global longitudinal strain (GLS) $\left(\Delta x^{2}=5.8, p=0.02\right)$ as well as to a clinical model including Karnofsky index and NT-proBNP $\left(\Delta X^{2}=6.2, p=0.01\right)$.

Conclusion: In patients with cardiac involvement in AL amyloidosis, systolic CVPD obtained from standard long axis cine views appear to indicate outcome better, when obtained in the anterior aortic plane (AAPSE) and provide incremental prognostic value to LGE and strain measurements.
\end{abstract}

Keywords: Cardiac valve plane excursion, Anterior aortic plane systolic excursion, AAPSE, MAPSE, TAPSE, AL amyloidosis, Risk assessment

\footnotetext{
* Correspondence: marco.ochs@med.uni-heidelberg.de

'Department of Cardiology, University of Heidelberg, INF 410, 69120

Heidelberg, Germany

Full list of author information is available at the end of the article
}

\section{) Biomed Central}

(c) The Author(s). 2017 Open Access This article is distributed under the terms of the Creative Commons Attribution 4.0 International License (http://creativecommons.org/licenses/by/4.0/), which permits unrestricted use, distribution, and reproduction in any medium, provided you give appropriate credit to the original author(s) and the source, provide a link to the Creative Commons license, and indicate if changes were made. The Creative Commons Public Domain Dedication waiver (http://creativecommons.org/publicdomain/zero/1.0/) applies to the data made available in this article, unless otherwise stated. 


\section{Background}

The early detection of myocardial protein infiltration is crucial to patient care in patients with suspected cardiac amyloidosis in systemic light chain amyloidosis (AL), as cardiac impairment determines overall prognosis and is present in up to $90 \%$ of patients during the course of the disease [1-4]. The median survival of affected patients with markedly elevated brain natriuretic peptide (BNP) and cardiac troponin levels is reduced to just about 8 months [5]. As renal insufficiency is common in AL and levels of serum biomarkers depend on glomerular filtration rate, imaging parameters are of special value in staging the disease.

Longitudinal systolic deformation of the left ventricle (LV) is known to be strongly associated with survival in AL amyloidosis [6-8]. Atrioventricular plane displacement contributes approximately $60 \%$ to the LV stroke volume [9]. The typical pattern of cardiac impairment follows a regional gradient from base to apex and is different in other causes of LV hypertrophy [10, 11]. Despite modern and more refined imaging technics, Mitral Annular Plane Systolic Excursion (MAPSE) has remained a widely accepted conventional parameter for rapid assessment of LV longitudinal function. MAPSE was introduced in the late 1980s [12] and was later applied for the evaluation of prognosis in heart failure patients [13]. However, recent findings have suggested an incremental prognostic value of Anterior Aortic Plane Systolic Excursion (AAPSE) by means of M-Mode echocardiography [14]. Systolic excursion patterns are heterogeneous [15] due to the differing passive regional resistance of the four interconnected fibrous rings and the active clockwise helical contraction of attached longitudinal myocardial fibers. Therefore, their predictive power of annular locations may also vary.

The purpose of the present study was a head-to-head comparison of the prognostic value of cardiovascular magnetic resonance (CMR) measurement locations for cardiac valve plane displacement (CVPD) in patients with AL amyloidosis.

\section{Methods}

\section{Study population and design}

We identified patients with cardiac biopsy-secured systemic AL amyloidosis who had undergone a CMR between February 2005 and October 2014 at our institution for a retrospective single-center post-hoc analysis. Patient selection is demonstrated in a flow chart (Fig. 1a). The mean interval between CMR and the start of chemotherapy was 23 days. The diagnosis was defined by the presence of monoclonal gammopathy by serum electrophoresis, immunofixation on serum and urine, and free light chain test, and confirmed by positive Congo red staining with birefringence under polarized light of any biopsy (periumbilical fat aspiration, rectum or target organ), positive immunohistology for kappa or lambda in the biopsy, and on the exclusion of hereditary forms of amyloidosis. The study population was referred to our imaging center for cardiologic work up with diagnosed light chain disorder. All 68 patients have signed a written informed consent for the retrospective analysis of clinical routine data. The use of de-identified patient data for research purposes was approved by the institutions ethics committee in accordance to the Declaration of Helsinki.

\section{CMR acquisition protocol and analysis of parameters}

All CMR examinations were performed on a 1.5 Tesla scanner (Achieva, Philips Healthcare, Best, The Netherlands) equipped with a cardiac phased array 32channel receiver coil. Cine images were obtained using a breath-hold segmented-k-space balanced fast-field echo sequence (bSSFP) employing retrospective electrocardiogram (ECG)- or pulse oximetric gating with 35 phases per cardiac cycle. Typical CMR imaging parameters were: field-of-view $(\mathrm{FOV})=350 \times 350 \mathrm{~mm}^{2}$, repetition time/echo time $(\mathrm{TR} / \mathrm{TE})=2.8 / 1.4 \mathrm{~ms}$, acquired voxel size $=2.2 \times 2.2 \times 8 \mathrm{~mm}^{3}$, flip angle $(\mathrm{FA})=60^{\circ}$, reconstructed voxel size $=1.3 \times 1.2 \times 8 \mathrm{~mm}^{3}$. Data were analyzed by a single examiner blinded to the patients' clinical status. Routine analysis was performed on a commercially available workstation (IntelliSpace Portal (ISP) Version 7.0.1, Philips Healthcare, Best, The Netherlands) with a semi-automatic software for volumetric analysis. Ventricular volumes, ejection fraction and LV myocardial mass were acquired in short axis stacks by manually tracing epi- and endocardial borders, excluding papillary muscles from the myocardium. Relative LGE mass was measured using a 5-standarddeviation (SD) threshold in reference to visually unenhanced myocardium (Fig. 1b) [16]. LGE was graduated into (1) none, (2) subendocardial or (3) transmural involvement (qualitative LGE). Global longitudinal strain (GLS) derived from cine SSFP of 2-, 3-, and 4-chamber long axis views by delineating endo- and epicardial borders at end-diastole using CVI $\mathrm{cmr}^{42}$ version 5.6.1, (Circle Cardiovascular Imaging, Calgary, Canada). Regional longitudinal strains are presented for basal (mean longitudinal strain of American Heart Association segments 1 to 6), midventricular (mean longitudinal strain of segments 7 to 12) and apical slices (mean longitudinal strain of segments 13 to 16 ).

\section{Assessment of cardiac valve plane displacement}

Regional systolic valve plane excursion was assessed at seven locations on anterior aortic plane systolic excursion (AAPSE), anterior, anterolateral, inferolateral, inferior and anterolateral mitral (MAPSE), and 
a

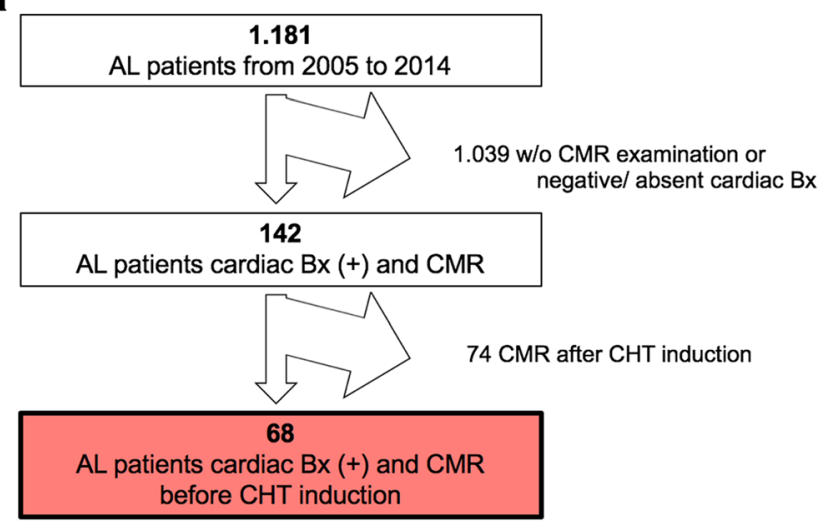

b
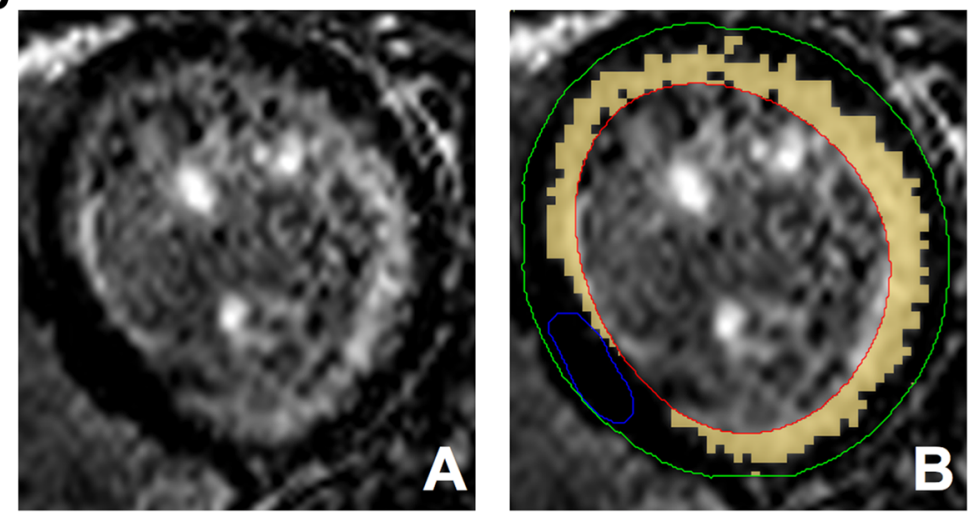

Fig. 1 a Patient selection flow chart. From 2005 to 2014 a total of 1.181 AL patients visited our institution. Patients had to be excluded for negative or absent cardiac biopsy (Bx), absent CMR examination, or CMR performed after induction of chemotherapy (CHT). The final study cohort consists of $68 \mathrm{AL}$ patients. b Late gadolinium enhancement (LGE) quantification by semiautomatic 5 SD-threshold selection. Contours for endo- (red) and epicardial borders (green) as well as for unenhanced reference myocardium (blue) were drawn manually. Signal intensities above 5-fold standard deviation of the reference myocardium were accounted to the LGE volume in relation to unenhanced myocardium (relative LGE, orange)

lateral tricuspid annulus (TAPSE) derived from cine bSSFP images in 2-, 3-, and 4-chamber views as previously described [15]. CVPD was defined as the distance from peak end-diastolic to peak end-systolic excursion of each annular region, where the endocardial insertion points of the aortic, mitral or tricuspid valve leaflets respectively served as anatomic landmarks.

\section{Blood samples}

Blood samples were drawn up to 14 days before the date of CMR examination. NT-proBNP (N-terminal prohormone of brain natriuretic peptide) and cTNT (cardiac troponin $\mathrm{T}$ ) were obtained from a commercially available fully automated analyzer based on a sandwich immunoassay (ELECSYS, Roche Diagnostics, Mannheim, Germany). Glomerular filtration rate was estimated by the "Modified Diet in Renal Diseases" (MDRD) formula.

\section{Follow-up}

Primary combined endpoint was all-cause mortality or heart transplantation. Follow-up was obtained by review of patient's electronic records or telephone interview with the patient or relative.

\section{Statistical methods}

Data were analyzed using MedCalc, version 15.11.4 (MedCalc Software, Ostend, Belgium). Continuous and normal distributed variables (Kolmogorov-Smirnov test, $p \geq 0.05)$ were expressed as mean \pm standard deviation. Group differences for continuous variables were tested using the independent t-test, and differences between nominal variables were assessed using the Fisher exact test. Continuous variables without 
normal distribution were stated as median and interquartile range (IQR), group differences were tested using the nonparametric Mann-Whitney $U$ test. Kaplan-Meier curves were used to estimate the distribution of survival as a function of the follow-up duration. Optimal cut-off values were defined by Receiver operating characteristics (ROC) and Youden's J statistic. The association of clinical, imaging and serological parameters with outcome was evaluated by uni- and multivariate Cox proportional-hazards regression models. Differences were considered statistically significant at $p<0.05$.

\section{Results}

Sixty-eight AL patients were included with a median follow up of 1.2 years (IQR, 0.3-4.1), of whom 44 $(64.7 \%)$ did not survive this period. Patients' characteristics are demonstrated in Table 1. Impairment of

Table 1 Baseline Characteristics. Patients with primary combined endpoint versus transplant-free survivors $(n=68)$

\begin{tabular}{|c|c|c|c|}
\hline Parameter & $\begin{array}{l}\text { Transplant-free Survivors } \\
(n=24)\end{array}$ & $\begin{array}{l}\text { Heart transplantation or death } \\
(n=44)\end{array}$ & $p$ \\
\hline Age (yrs) & $57 \pm 8$ & $59 \pm 11$ & n.s. \\
\hline Male $(n)^{a}$ & $13(54 \%)$ & $27(61 \%)$ & n.s. \\
\hline BMI $\left(\mathrm{kg} / \mathrm{m}^{2}\right)$ & $25(23-27)$ & $25(23-27)$ & n.s. \\
\hline Heart rate (bpm) & $78 \pm 13$ & $80 \pm 14$ & n.s. \\
\hline NYHA class & $2(2-3)$ & $3(2-3)$ & 0.03 \\
\hline Karnofsky Index & $85 \pm 6$ & $76 \pm 10$ & 0.0001 \\
\hline \multicolumn{4}{|l|}{ Laboratory data } \\
\hline $\operatorname{MDRD}\left(\mathrm{mL} / \mathrm{min} / 1.73 \mathrm{~m}^{2}\right)$ & $81 \pm 17$ & $83 \pm 14$ & n.s. \\
\hline NT-proBNP (pg/mL) & $1177(528-3249)$ & $3243(1157-7505)$ & 0.01 \\
\hline cTNT ULN (n) ${ }^{a}$ & $8(38 \%)$ & $18(41 \%)$ & n.s. \\
\hline FLC difference (mg/dL) & $1,1130(435-5435)$ & 3351 (975-8971) & n.s. \\
\hline \multicolumn{4}{|l|}{ CMR parameters } \\
\hline AAPSE (mm) & $8.8(6.9-10.4)$ & $6.1(4.6-9.4)$ & 0.02 \\
\hline MAPSE anterior (mm) & $8.1(5.3-10.0)$ & $6.2(4.1-8.6)$ & n.s. \\
\hline MAPSE anterolateral (mm) & $10.5(8.1-13.4)$ & $7.3(5.4-11.7)$ & 0.03 \\
\hline MAPSE inferolateral (mm) & $9.3(6.3-12.1)$ & $7.6(5.2-12.2)$ & n.s. \\
\hline MAPSE inferior (mm) & $9.2(7.4-11.1)$ & $8.0(5.0-11.7)$ & n.s. \\
\hline MAPSE inferoseptal (mm) & $7.6(6.1-10.4)$ & $7.1(4.5-10.8)$ & n.s. \\
\hline TAPSE (mm) & $18.0(10.0-22.4)$ & $14.1(9.0-19.7)$ & n.s. \\
\hline GLS (\%) & $-14.8 \pm 5.1$ & $-13.2 \pm 5.2$ & n.s. \\
\hline$L S_{\text {basal }}(\%)$ & $-12.3 \pm 6.1$ & $-11.4 \pm 8.5$ & n.s. \\
\hline$L S_{\text {mid }}(\%)$ & $-16.4 \pm 5.2$ & $-14.7 \pm 5.5$ & n.s. \\
\hline $\mathrm{LS}_{\text {apical }}(\%)$ & $-17.6 \pm 5.9$ & $-16.2 \pm 6.6$ & n.s. \\
\hline Ejection Fraction (\%) & $62 \pm 10$ & $55 \pm 13$ & 0.03 \\
\hline Septal wall (mm) & $14 \pm 5$ & $13 \pm 3$ & n.s. \\
\hline Lateral wall (mm) & $10 \pm 4$ & $10 \pm 4$ & n.s. \\
\hline Relative LV Mass $\left(\mathrm{g} / \mathrm{m}^{2}\right)$ & $75 \pm 21$ & $73 \pm 35$ & n.s. \\
\hline $\mathrm{LA}(\mathrm{mm})$ & $39 \pm 7$ & $42 \pm 7$ & n.s. \\
\hline Relative LGE (\%) & $16.5(10.0-35.7)$ & $25.0(18.1-30.4)$ & n.s. \\
\hline Qualitative LGE (n) & $2(2-3)$ & $2(2-3)$ & n.s. \\
\hline
\end{tabular}

Normal distributed variables are presented as mean \pm SD, non-parametric distributions are shown as Median (1st quartile-3rd quartile). ${ }^{a}$ dichotomous variable $B M I$ body mass index, MDRD glomerular filtration rate calculated with the formula of "Modification of Diet in Renal Disease", NT-proBNP N-terminal prohormone of brain natriuretic peptide, CTNT ULN cardiac troponin T upper limit of normal, FLC free light chain difference, AAPSE anterior aortic plane systolic excursion, MAPSE mitral annular plane systolic excursion, TAPSE tricuspid annular plane systolic excursion, GLS global longitudinal strain, $L S_{b a s a l}$ mean longitudinal strain of basal segments $1-6, L S_{\text {mid }}$ mean longitudinal strain of midventricular segments $7-12, L S_{\text {apical }}$ mean longitudinal strain of apical segments $13-16, L A$ left atrium, relative LGE proportion of late gadolinium enhancement quantified by 5SD method, qualitative LGE Late Gadolinium Enhancement graduated in (1) none, (2) subendocardial or (3) transmural involvement 
NYHA functional class (3 (IQR, 2-3) vs 2 (IQR, 2-3); $p=0.001)$ and Karnofsky index $(85 \pm 6$ vs. $76 \pm 10$; $p=0.0001)$ were both accentuated in patients with primary endpoint. Regarding established serum biomarkers, only NT-proBNP showed significant differences between transplant-free survivors and patients with primary endpoint (1177 pg/mL (IQR, 528-3249) vs $3243 \mathrm{pg} / \mathrm{mL}$ (IQR, 1157-7505); $p=0.01$ ). Among all annular locations, only AAPSE $(8.8 \mathrm{~mm}$ (IQR, 6.9-10.4) vs $6.1 \mathrm{~mm}$ (IQR, 4.6-9.4); $p=0.02$ ) and MAPSE $_{\text {anterolateral }}(10.5 \mathrm{~mm} \quad(\mathrm{IQR}, \quad 8.1-13.4) \quad$ vs $7.3 \mathrm{~mm}$ (IQR, 5.4-11.7); $p=0.03$ ) were significantly reduced in patients who died or were heart transplanted during the follow up period.

AAPSE $\left(X^{2}=15.6 ; p=0.0002\right)$ was found the best predictor of transplant-free survival compared to all other valvular plane locations as well as relative/ qualitative LGE, GLS or regional longitudinal strain in univariate Cox-regression analysis (Fig. 2; Table 2). The slice-wise assessment of regional longitudinal strain did not reveal a significant benefit over GLS in terms of predictive power (Table 2). AAPSE $\left(\mathrm{X}^{2}=21.6, p=0.0001\right)$ was also found beneficial for predicting a combined endpoint of cardiac death, heart transplantation, and hospitalization compared to other established parameters (GLS: $\mathrm{X}^{2}=11.4$, $p=0.0007$; relative LGE: $\left.\mathrm{X}^{2}=4.4, p=0.03\right)$.

An optimized cut-off value for AAPSE of $\leq 7.6 \mathrm{~mm}$ was calculated to predict whether patients would reach a primary endpoint within 6 months from index examination (AUC $=0.80 ; \mathrm{CI}: 0.68$ to 0.89 ; $p<0.0001)$. The Kaplan-Meier survival curve is demonstrated in Fig. 3 (log-rank $p \leq 0.0001)$. Median survival of patients with AAPSE $\leq 7.6 \mathrm{~mm}$ was 0.5 years (IQR, 0.2-2.1) compared to 3.9 years (IQR, 1.5-5.8).

To further evaluate the incremental predictive power of AAPSE for transplant-free survival, a sequential Coxregression was performed for a clinical (NT-proBNP and Karnofsky Index; $\left.\chi^{2}=15.4\right)$ and an imaging prediction model (LGE and GLS; $X^{2}=10.2$ ) (Fig. 4). Hereby, the stepwise addition of AAPSE provided a significant increase in predictive power for both models (clinical: AAPSE $\Delta \mathrm{X}^{2}=6.2, p=0.01$; imaging: AAPSE $\Delta \mathrm{X}^{2}=5.8$, $p=0.02)$.

\section{Discussion}

Our data indicate that in a head-to-head comparison in patients with biopsy-proven cardiac involvement in AL amyloidosis, the AAPSE appears to better predict outcome than measurements in other regions such as the mitral or tricuspid valve ring with incremental value to established clinical, biochemical, and imaging prognosticators.

The measurement of CVPD is traditionally reduced to MAPSE and TAPSE, which are both obtained in 4chamber view on the lateral or septal mitral and on the lateral tricuspid annular location respectively [12]. However, recent findings have raised the hypothesis for significant heterogeneities of the prognostic value at different locations on the cardiac valve plane [14]. Therefore, the present data provides a comprehensive comparison and suggests the anterior aortic location (AAPSE) is associated with a better risk prediction in

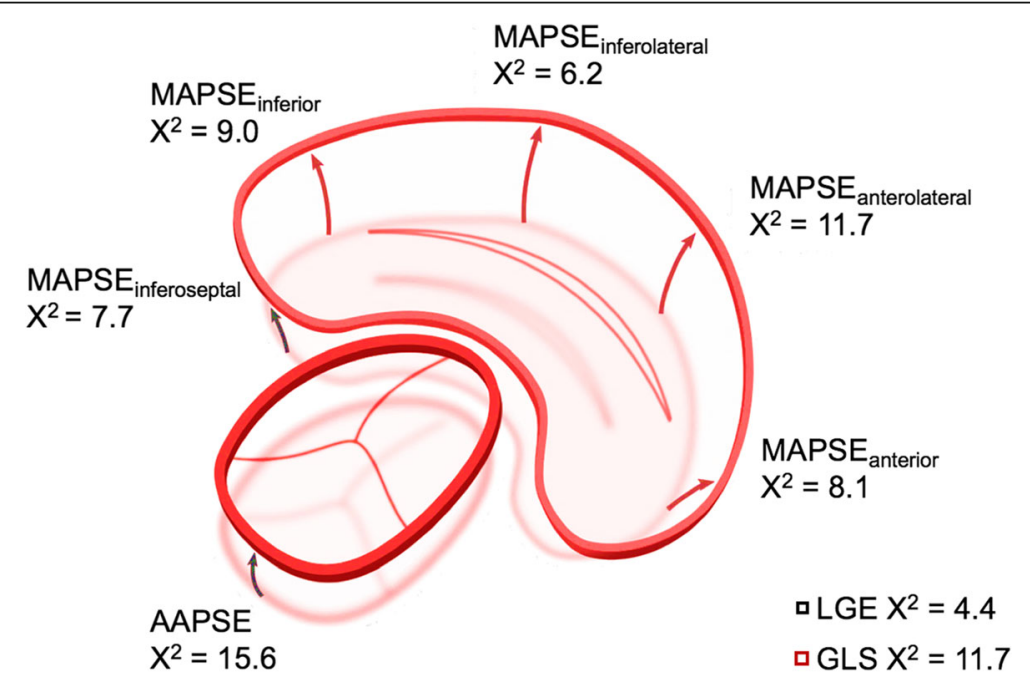

Fig. 2 Distribution of the prognostic value for cardiac valve plane displacement on varying locations. Chi-square $\left(X^{2}\right)$ resulted from univariate regression analyses. To relate the prognostic value of cardiac valve plane motion with established predictors in $\mathrm{AL}$ amyloidosis relative LLGE and global longitudinal strain (GLS) are presented 
Table 2 Univariate Cox-regression for overall survival or heart transplantation $(n=68)$

\begin{tabular}{|c|c|c|c|c|}
\hline Variable & $x^{2}$ & $\mathrm{HR}$ & $95 \% \mathrm{Cl}$ & $p$ \\
\hline NYHA class & 7.5 & 1.75 & 1.15 to 2.68 & 0.001 \\
\hline Karnofsky Index & 21.3 & 0.91 & 0.88 to 0.95 & 0.0001 \\
\hline \multicolumn{5}{|l|}{ CMR parameters } \\
\hline AAPSE (mm) & 15.6 & 0.10 & 0.03 to 0.34 & 0.0002 \\
\hline MAPSE anterior (mm) & 8.1 & 0.19 & 0.06 to 0.60 & 0.004 \\
\hline MAPSE anterolateral (mm) & 11.7 & 0.23 & 0.10 to 0.56 & 0.001 \\
\hline MAPSE inferolateral (mm) & 6.2 & 0.36 & 0.16 to 0.83 & 0.02 \\
\hline MAPSE inferior (mm) & 9.0 & 0.26 & 0.10 to 0.63 & 0.003 \\
\hline MAPSE inferoseptal (mm) & 7.7 & 0.26 & 0.10 to 0.68 & 0.01 \\
\hline TAPSE (mm) & 6.7 & 0.94 & 0.90 to 0.99 & 0.01 \\
\hline GLS (\%) & 11.7 & 1.12 & 1.05 to 1.19 & 0.0006 \\
\hline $\mathrm{LS}_{\text {basal }}(\%)$ & 8.3 & 1.08 & 1.02 to 1.13 & 0.006 \\
\hline$L S_{\text {mid }}(\%)$ & 12.8 & 1.13 & 1.06 to 1.21 & 0.0005 \\
\hline $\mathrm{LS}_{\text {apical }}(\%)$ & 6.9 & 1.07 & 1.02 to 1.12 & 0.005 \\
\hline Ejection Fraction (\%) & 11.6 & 0.95 & 0.93 to 0.98 & 0.0007 \\
\hline Septal wall (mm) & 0.7 & 1.03 & 0.96 to 1.11 & n.s. \\
\hline Relative LV Mass $\left(\mathrm{g} / \mathrm{m}^{2}\right)$ & 1.8 & 1.01 & 0.99 to 1.02 & n.s. \\
\hline LA dilation $^{a}$ & 0.02 & 1.05 & 0.55 to 1.97 & n.s. \\
\hline Relative LGE (\%) & 4.4 & 1.02 & 1.00 to 1.04 & 0.03 \\
\hline Qualitative LGE (n) & 6.8 & 1.50 & 1.09 to 2.06 & 0.009 \\
\hline \multicolumn{5}{|l|}{ Laboratory data } \\
\hline CTNT ULN $^{a}$ & 5.3 & 1.94 & 1.09 to 3.45 & 0.02 \\
\hline log NT-proBNP (pg/mL) & 13.0 & 1.52 & 1.20 to 1.94 & 0.0006 \\
\hline $\operatorname{MDRD}\left(\mathrm{mL} / \mathrm{min} / 1.73 \mathrm{~m}^{2}\right)$ & 0.2 & 1.00 & 0.99 to 1.01 & n.s. \\
\hline log FLC difference (mg/dL) & 6.3 & 1.71 & 1.10 to 2.65 & 0.02 \\
\hline
\end{tabular}

$X^{2}$ chi square, $H R$ hazard ratio, $\mathrm{Cl}$ confidence interval, ${ }^{a}$ dichotomous variable AAPSE anterior aortic plane systolic excursion, MAPSE mitral annular plane systolic excursion, TAPSE tricuspid annular plane systolic excursion, GLS global longitudinal strain, $L S_{\text {basal }}$ mean longitudinal strain of basal segments $1-6, L S_{\text {mid }}$ mean longitudinal strain of midventricular segments $7-12, L S_{\text {apical }}$ mean longitudinal strain of apical segments 13-16, LA left atrium, relative LGE proportion of late gadolinium enhancement quantified by SSD method, qualitative LGE late gadolinium enhancement graduated in (1) none, (2) subendocardial or (3) transmural involvement, CTNT ULN cardiac troponin T upper limit of normal, NT-proBNP N-terminal prohormone of brain natriuretic peptide, MDRD glomerular filtration rate calculated with the formula of "Modification of Diet in Renal Disease", FLC free light chain difference

patients with $\mathrm{AL}$ amyloidosis. This finding cannot be easily explained, also because the motion of the aortic annulus is driven by a complex interplay between inserting myocardial fibers that are clockwise descending towards the apex and counteracting annular and aortic stiffness. Beyond risk prediction, future studies will have to address a potential role of AAPSE to indicate early myocardial manifestation or therapeutic benefits by guiding chemotherapeutic regimens in AL amyloidosis.

At present the most widely used staging system and validated gold standard to estimate the severity of cardiac impairment and life expectancy [17] is based on serum biomarkers NT-proBNP and cTNT [5], but serum levels depend on glomerular filtration rate, which is commonly affected in AL. Therefore, cardiac imaging parameters are gaining importance and our data provide further evidence that AAPSE may have an incremental predictive power to preexisting staging systems. As atrioventricular valve plane motion reflects the longitudinal shortening of the left ventricle, previous observations also support the significance of its impairment in AL. The relative longitudinal axes shortening (LAS) and global longitudinal strain (GLS) are both independent predictors of survival $[6,8]$. Other innovative risk predictors in amyloidosis have emerged in cardiac magnetic resonance imaging, including late gadolinium enhancement, T1 mapping, and extracellular volume (ECV) which allow for a non-invasive semi-quantitative assessment of amyloid infiltration [18] and correlate with allcause mortality [13, 19-21]. Recently, the prognostic value of left atrial function in AL was emphasized [22]. Nevertheless, there are obvious advantages of cardiac valve plane measurements over more refined imaging techniques for assessment of individual prognosis.

First, quantification is less time consuming than the post-processing and analysis of other approaches. Regarding particularly strain measurements, the presence of non-standardized tracking algorithms leads to variable results between different software solutions and insufficient tracking is a common source of errors. These issues have been demonstrated previously and are the main limitation for segmental analysis of longitudinal strain by feature tracking in CMR [23]. Moreover, the present data does not encourage for a routine assessment of slice-wise regional longitudinal strains as a significant benefit over GLS in terms of prognostication in AL amyloidosis could not be found. Second, measurements can be taken from conventional long axis views and don't rely on additional sequences. Third, a noncontrast protocol avoids potential, albeit small risks associated with the administration of gadolinium chelates such as systemic nephrogenic fibrosis and gadolinium deposition in the human brain [24, 25]. Facing these issues, cardiac valve plain motion appears to allow a fast, cost-effective and reliable evaluation of prognosis. AAPSE may better predict mortality than global longitudinal strain according to the present analysis. This finding suggests, that differentiation of regional longitudinal function might be favorable over measurements of global longitudinal function for risk assessment. The typical pattern follows a gradient of impairment from basal to apical segments, which is usually termed as "apical sparing" $[10,11]$. The fact that longitudinal shortening is the main contributor to left ventricular ejection fraction, might serve as an explanation why this contractile 


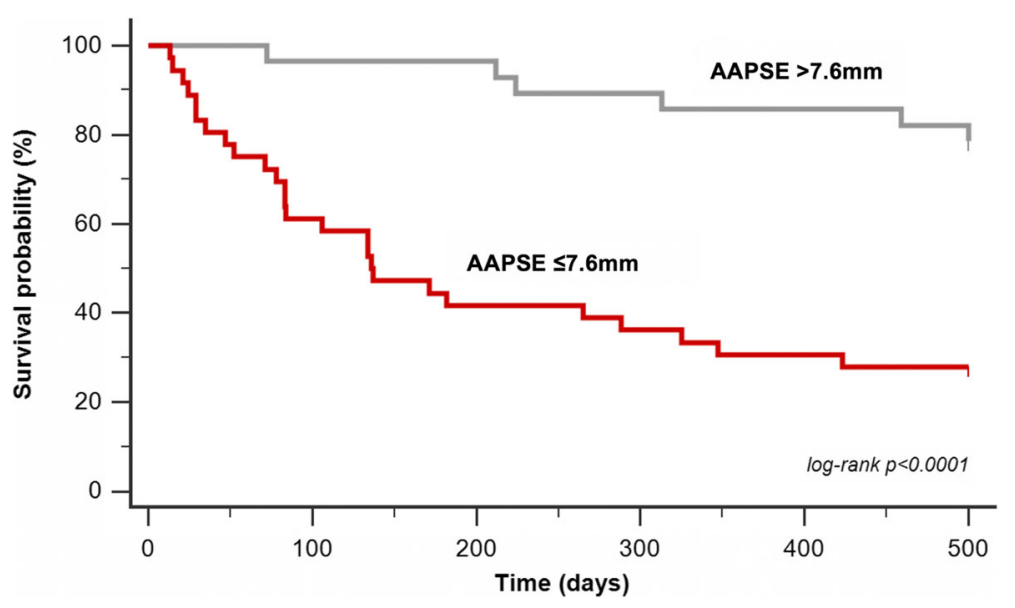

Fig. 3 Kaplan-Meier survival curve demonstrates the discriminative prognostic value of AAPSE in cardiac biopsy proven AL amyloidosis. Cut-off (AAPSE $\leq 7.6 \mathrm{~mm}$ ) was calculated by ROC analyses (AUC $=0.80 ; \mathrm{Cl}$ : 0.68 to $0.89 ; p<0.0001$ ) and Youden's-J statistic to predict all-cause death or heart transplantation within 6 months from index examination $(n=68)$

component is more sensitive to pathologic changes [9, 26], and eventually prognosis.

CVPD measurements were initially adopted from MMode echocardiography. Since then, several methods of measurement have been proposed [22, 27-30], while no standard technique has been recommended by official guidelines. The present analysis relies on a mode of measurement, that has previously demonstrated a good reproducibility between observers, studies, and modalities [15].

The present study has some limitations. 1) The data were obtained retrospectively and have to be confirmed in later, prospective samples. 2) The study was performed at a single-center and was restricted to patients with AL amyloidosis, which limits its generalizability. 3) The study cohort might be selection biased excluding patients unable to perform a CMR examination with rest dyspnea, severe arrhythmias, or claustrophobia.

\section{Conclusion}

The predictive value of cardiac valve plane excursion varies between different annular locations. The Anterior Aortic Plane Systolic Excursion (AAPSE) appears to best
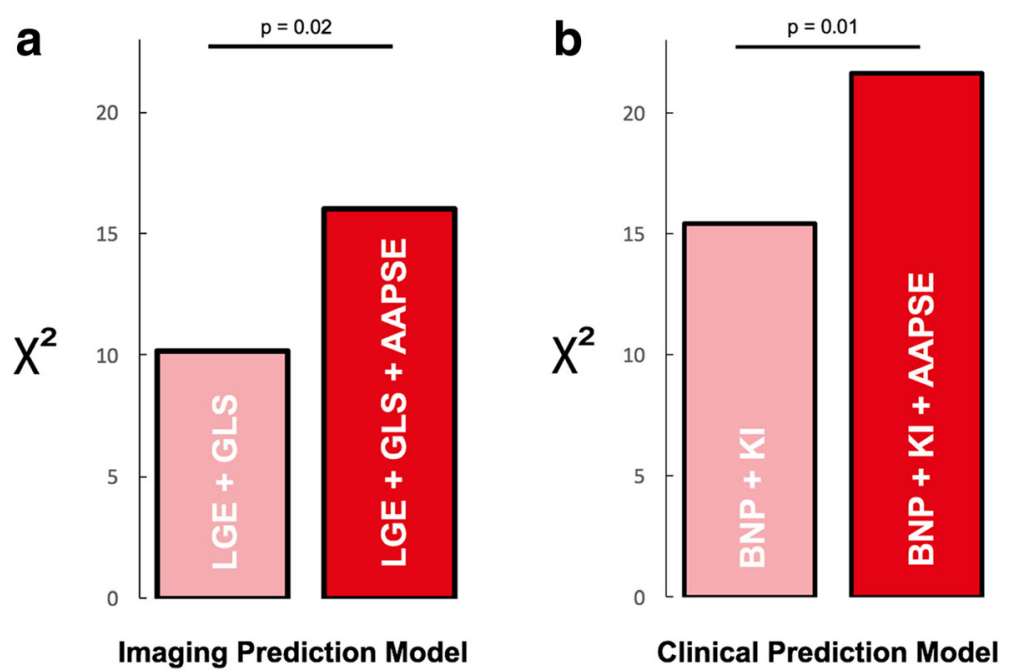

Fig. 4 Incremental prognostic power of AAPSE. Sequential Cox-regression analysis was performed to evaluate the incremental predictive power of AAPSE for transplant-free survival in an (a) imaging (LGE and GLS; $\left.X^{2}=10.2\right)$ and a (b) clinical prediction model (NT-proBNP and Karnofsky Index; $x^{2}=15.4$ ). AAPSE significantly improved the predictive power of both models (imaging: AAPSE $\Delta x^{2}=5.8, p=0.02$; clinical: AAPSE $\left.\Delta x^{2}=6.2, p=0.01\right)$ 
predict all-cause mortality or heart transplantation in systemic AL amyloidosis with incremental prognostic value to established clinical, biochemical, and imaging prognosticators.

\begin{abstract}
Abbreviations
AAPSE: Anterior aortic plane systolic excursion; AL: Systemic light chain amyloidosis; BNP: Brain natriuretic peptide; bSSFP: Balanced steady state free precession; CMR: Cardiovascular magnetic resonance; CVPD: Cardiac valve plane displacement; GLS: Global longitudinal strain; IQR: Interquartile range; LGE: Late gadolinium enhancement; LV: Left ventricle/left ventricular; MAPSE: Mitral annular plane systolic excursion; TAPSE: Tricuspid annular plane systolic excursion.
\end{abstract}

\section{Acknowledgements}

We thank our technologists for their excellent support.

\section{Funding}

The present study was not financially supported.

\section{Availability of data and materials}

The datasets used and/or analysed during the current study are available from the corresponding author on reasonable request.

\section{Authors' contributions}

$\mathrm{MO}$ responsible for conception of study design, data acquisition and analysis, statistical assessment, and writing of the manuscript. TF involved in data acquisition and analysis, responsible for implementation and development of image analysis software. NA involved in data acquisition and analysis. JR involved in drafting of the manuscript and data analysis. FA involved in drafting of the manuscript, data analysis, and statistical review. DM involved in conception of study design. FS involved in data acquisition and analysis. $\mathrm{UH}$ involved in conception of study design, data acquisition, and drafting of the manuscript. SS involved in conception of study design, data acquisition, and drafting of the manuscript. HK involved in drafting of the manuscript, final approval of the manuscript submitted. MFA involved in interpretation of the data and critical review of the manuscript. SJB involved in conception and design, analysis and interpretation of data and in drafting of the manuscript. All authors read and approved the final manuscript.

\section{Ethics approval and consent to participate}

The analysis of de-identified patient data was approved by the institutions ethics committee (reference number: S-154/2015, Ethikkommission Heidelberg, Alte Glockengießerei 11/1, 60,115 Heidelberg). Documentation of consent was waived for this retrospective study.

\section{Consent for publication}

Documentation of consent was waived for this retrospective study, which utilized de-identified patient data.

\section{Competing interests}

The authors declare that they have no competing interests.

\section{Publisher's Note}

Springer Nature remains neutral with regard to jurisdictional claims in published maps and institutional affiliations.

\section{Author details \\ 'Department of Cardiology, University of Heidelberg, INF 410, 69120 Heidelberg, Germany. ${ }^{2}$ Department of Hematooncology, University of Heidelberg, INF 410, 69120 Heidelberg, Germany.}

Received: 8 February 2017 Accepted: 16 October 2017

Published online: 09 November 2017

\section{References}

1. Dubrey SW, Cha K, Anderson J, Chamarthi B, Reisinger J, Skinner M, et al. The clinical features of immunoglobulin light-chain (AL) amyloidosis with heart involvement. QJM. 1998;91:141-57.
2. Selvanayagam JB, Hawkins PN, Paul B, Myerson SG, Neubauer S. Evaluation and management of the cardiac amyloidosis. J Am Coll Cardiol. 2007;50: 2101-10.

3. Sipe JD, Benson MD, Buxbaum JN, Ikeda S-I, Merlini G, Saraiva MJM, et al. Amyloid fibril protein nomenclature: 2010 recommendations from the nomenclature committee of the international society of Amyloidosis. Amyloid. 2010;17:101-4. Available from: http://www.ncbi.nlm.nih.gov/ pubmed/21039326.

4. Kristen AV, Perz JB, Schonland SO, Hegenbart U, Schnabel PA, Kristen JH, et al. Non-invasive predictors of survival in cardiac amyloidosis. Eur J Heart Fail. 2007;9:617-24. Available from: http://www.ncbi.nlm.nih.gov/pubmed/ 17350331.

5. Dispenzieri A, Gertz MA, Kyle RA, Lacy MQ, Burritt MF, Therneau TM, et al. Serum cardiac troponins and N-terminal pro-brain natriuretic peptide: a staging system for primary systemic amyloidosis. J Clin Oncol. 2004;22: 3751-7. Available from: http://jco.ascopubs.org/content/22/18/3751.full.

6. Buss SJ, Emami M, Mereles D, Korosoglou G, Kristen AV, Voss A, et al. Longitudinal left ventricular function for prediction of survival in systemic light-chain amyloidosis: incremental value compared with clinical and biochemical markers. J Am Coll Cardiol. 2012;60:1067-76. Available from: http://doi.org/10.1016/j.jacc.2012.04.043.

7. Koyama J, Davidoff R, Falk RH. Longitudinal myocardial velocity gradient derived from pulsed Doppler tissue imaging in AL amyloidosis: a sensitive indicator of systolic and diastolic dysfunction. 2004;17:36-44. Available from: http://www.sciencedirect.com/science/article/pii/S0894731703008149

8. Riffel JH, Mereles D, Emami M, Korosoglou G, Kristen AV, Aurich M, et al. Prognostic significance of semiautomatic quantification of left ventricular long axis shortening in systemic light-chain amyloidosis. Amyloid. 2015;22: 45-53. Available from: http://informahealthcare.com/doi/abs/10.3109/ 13506129.2014.992515.

9. Carlsson M, Ugander M, Mosén H, Buhre T, Arheden H. Atrioventricular plane displacement is the major contributor to left ventricular pumping in healthy adults, athletes, and patients with dilated cardiomyopathy. AJP: Heart Circ Physiol. 2007;292:H1452-9. Available from: http://www.ncbi.nlm. nih.gov/pubmed/17098822.

10. Koyama J, Ray-Sequin PA, Falk RH. Longitudinal myocardial function assessed by tissue velocity, strain, and strain rate tissue Doppler echocardiography in patients with AL (primary) cardiac amyloidosis. Circulation. 2003;107:2446-52. Available from: http://circ.ahajournals.org/ content/107/19/2446.short.

11. Phelan D, Collier P, Thavendiranathan P, Popović ZB, Hanna M, Plana JC, et al. Relative apical sparing of longitudinal strain using two-dimensional speckle-tracking echocardiography is both sensitive and specific for the diagnosis of cardiac amyloidosis. Heart. 2012;98:1442-8.

12. Pai RG, Bodenheimer MM, Pai SM, Koss JH, Adamick RD, Koss H. Usefulness of systolic excursion of the mitral anulus as an index of left ventricular systolic function. Am J Cardiol. 1991;67:222-4. Available from: http://www. sciencedirect.com/science/article/pii/000291499190453R.

13. Syed IS, Glockner JF, Feng D, Araoz PA, Martinez MW, Edwards WD, et al. Role of cardiac magnetic resonance imaging in the detection of cardiac amyloidosis. J Am Coll Cardiol Img. 2010;3:155-164. Elsevier Inc.

14. Ochs MM, Riffel J, Kristen AV, Hegenbart U, Schönland S, Hardt SE, et al. Anterior Aortic Plane Systolic Excursion: A Novel Indicator of Transplant-Free Survival in Systemic Light-Chain Amyloidosis. J Am Soc Echocardiogr. Official Publication of the American Society of Echocardiography 2016; 29(12):1188-1196. http://doi.org/10.1016/j.echo.2016.09.003.

15. Ochs MM, Fritz T, Andre F, Riffel J, Mereles D, Müller-Hennessen M, et al. A comprehensive analysis of cardiac valve plane displacement in healthy adults: age-stratified normal values by cardiac magnetic resonance. Int J Cardiovasc Imaging. 2017;33(5):721-729. http://doi.org/10.1007/s10554-016-1058-y.

16. Moravsky G, Ofek E, Rakowski H, Butany J, Williams L, Ralph-Edwards A, et al. Myocardial fibrosis in hypertrophic Cardiomyopathy. J Am Coll Cardiol Img. 2013;6:587-96.

17. Gertz MA. Immunoglobulin light chain amyloidosis: 2014 update on diagnosis, prognosis, and treatment. Am J Hematol. 2014;89:1132-40. Available from: http://www.ncbi.nlm.nih.gov/pubmed/25407896.

18. Hashimura H, Ishibashi-Ueda H, Yonemoto $\mathrm{Y}$, Ohta-Ogo K, Matsuyama TA, Ikeda $Y$, et al. Late gadolinium enhancement in cardiac amyloidosis: attributable both to interstitial amyloid deposition and subendocardial fibrosis caused by ischemia. Heart Vessels. 2016;31:990-5. https://doi.org/10. 1007/s00380-015-0658-0. 
19. Boynton SJ, Geske JB, Dispenzieri A, Syed IS, Hanson TJ, Grogan M, et al. LGE provides incremental prognostic information over serum biomarkers in AL cardiac Amyloidosis. J Am Coll Cardiol Img. 2016;9:680-6. Available from: http://www.sciencedirect.com/science/article/pii/S1936878X16301917.

20. Maceira AM, Prasad SK, Hawkins PN, Roughton M, Pennell DJ. Cardiovascular magnetic resonance and prognosis in cardiac amyloidosis. J Cardiovasc Magn Reson. 2008;10:54. Available from: https://doi.org/10.1186/1532-429X10-54.

21. Fontana M, Pica S, Reant P, Abdel-Gadir A, Treibel TA, Banypersad SM, et al. Prognostic value of late gadolinium enhancement cardiovascular magnetic resonance in cardiac Amyloidosis. Circulation. 2015;132:1570-9.

22. Garg P, Kidambi A, Ripley DP, Dobson LE, Swoboda PP, Musa TA, et al. Mitral annular plane systolic excursion and intra-myocardial haemorrhage in acute myocardial infarction. J Cardiovasc Magn Reson. 2015;17:1. https://doi. org/10.1186/1532-429X-17-S1-P163.

23. Wu L, Germans T, Güçlü A, Heymans MW, Allaart CP, van Rossum AC. Feature tracking compared with tissue tagging measurements of segmental strain by cardiovascular magnetic resonance. J Cardiovasc Magn Reson; 2014;16:10.

24. Radbruch A, Weberling LD, Kieslich PJ, Eidel O, Burth S, Kickingereder P, et al. Gadolinium retention in the dentate nucleus and globus pallidus is dependent on the class of contrast agent. Radiology. 2015;275:783-91. Available from: http://pubs.rsna.org/doi/10.1148/radiol.2015150337.

25. Kanda T, Ishii K, Kawaguchi H, Kitajima K, Takenaka D. High signal intensity in the dentate nucleus and globus pallidus on unenhanced T1-weighted MR images: relationship with increasing cumulative dose of a gadoliniumbased contrast material. Radiology. 2014;270:834-41. Available from: http:/ pubs.rsna.org/doi/10.1148/radiol.13131669.

26. Carlhäll C, Wigström L, Heiberg E, Karlsson M, Bolger AF, Nylander E. Contribution of mitral annular excursion and shape dynamics to total left ventricular volume change. AJP: Heart Circ Physiol. 2004;287:H1836-41.

27. Maceira AM, Prasad SK, Khan M, Pennell DJ. Normalized Left Ventricular Systolic and Diastolic Function by Steady State Free Precession Cardiovascular Magnetic Resonance. J Cardiovasc Magn Reson. 2006;8(3): 417-426. http://doi.org/10.1080/10976640600572889.

28. Saba SG, Chung S, Bhagavatula S, Donnino R, Srichai MB, Saric M, et al. A novel and practical cardiovascular magnetic resonance method to quantify mitral annular excursion and recoil applied to hypertrophic cardiomyopathy. J Cardiovasc Magn Reson. 2014;16:35. Available from: http://www. pubmedcentral.nih.gov/articlerender.fcgi?artid=4041905\&tool= pmcentrez\&rendertype $=$ abstract.

29. Bulluck H, Ngamkasem H, Sado D, Treibel TA, Fontana M, Maestrini V, et al. A simple technique to measure TAPSE and MAPSE on CMR and normal values. J Cardiovasc Magn Reson. 2014;16:1.

30. Abdel-Aty H, Katus HA, Lehrke S, Steen H. CMR derived MAPSE and TAPSE measurements in hypertrophic cardiomyopathy: comparison to healthy volunteers. J Cardiovasc Magn Reson. 2012;14:1.

\section{Submit your next manuscript to BioMed Central and we will help you at every step:}

- We accept pre-submission inquiries

- Our selector tool helps you to find the most relevant journal

- We provide round the clock customer support

- Convenient online submission

- Thorough peer review

- Inclusion in PubMed and all major indexing services

- Maximum visibility for your research

Submit your manuscript at www.biomedcentral.com/submit

) Biomed Central 